begreiflicherweise eine Unzahl ungelöster oder scheinbar unlösbarer Probleme zur Folge hatten. Erst durch die Veröffentlichung von A. Bögli über die Mischungskorrosion wurde zum Beispiel klar, daß sich die großen Höhlen nicht von außen nach innen entwickeln, sondern umgekehrt. Durch sie wurde auch die rätselhafte Kalklösung bei gänzlicher Wasserfüllung, also bei fehlender $\mathrm{CO}_{2}-Z$ ufuhr, erklärt. (Bögli : Mischungskorrosion. Ein Beitrag zum Verkarstungsproblem; «Erdkunde», Archiv für wissenschaftliche Geographie, Bd. XVIII, Lfg. 2, Bonn 1964.)

Die Höhlenkunde bedient sich vieler Wissenschaften, die für die besonderen Verhältnisse der Höhlen nicht selten Spezialgebiete entwickelt haben; Karsthydrologie, Speläobiologie und Höhlenmeteorologie sind einige Beispiele. Der Einsatz dieser Wissenszweige verrät deutlich, daß wir es in der Speläologie nicht nur mit einer analysierenden, sondern auch mit einer synthetisierenden Wissenschaft zu tun haben. Der Arbeitsbereich der Speläologie ist, auf der Grundlage der Geomorphologie, eine Synthese aller mit der Höhle verbundener Eigenschaften. Da der Synthese aber als Weg zum Erkennen und Verstehen der Einzelerscheinungen eine Analyse vorausgehen muß, so kann diese Wissenschaft der Grundlagenforschung, vor allem im Bereiche der Morphologie und der Karsthydrologie, nicht entraten. Trotzdem liegt das Endziel der Speläologie nicht darin, sondern im lückenlosen Erfassen einer Landschaft im geographischen Sinne, der Höhlenlandschaft. Infolge der Seltenheit menschlicher Eingriffe ist diese eine Naturlandschaft, was an der Gesamtkonzeption jedoch nichts ändert. Die Speläologie ist ihrem ganzen Wesen nach nicht nur ein Teil der Geomorphologie, sondern darüber hinaus eine geographische Wissenschaft.

Legende zu rechtsstehendem Farbbild:

Sinterfahnen im Medusendom des Hölloches. Sie bestehen aus reinem Kalzit und sind so dünn, $\mathrm{da} ß$ das Licht hindurchleuchtet.

\title{
DIE GEOGRAPHIE ALS KONZENTRATIONSFACH IN SCHULKOLONIEN
}

\author{
KURT BÖSIGER
}

\section{GEOGRAPHISCHE ARBEITSGEMEINSCHAFTEN AN DER MITTELSCHULE}

Noch vor 30 Jahren steckten die Schulkolonien sowohl in organisatorischer wie prinzipieller Hinsicht im Versuchsstadium (Lit. 1). Denn nur mit der Veränderung des Ortes - unter Beibehaltung der in der Schulstube üblichen Unterrichtsgestaltung sind längst nicht alle Möglichkeiten ausgeschöpft. Annaheim versuchte schon im Sommer 1935 «das Gemeinschaftsprinzip für das gesamte Leben der Kolonie einschließlich der inhaltlichen Ausprägung der Unterrichtsarbeit von Bedeutung werden zu lassen». Er wählte für seine 4. Sekundarklasse (achtes Schuljahr) die Dorfgemeinschaft von Feldis (GR), also den Alpenbauern als Nutzer und Gestalter der Naturlandschaft, als Schwerpunktthema seines Gesamtunterrichtes. Seither ist der unschätzbare Wert solcher Arbeitsgemeinschaften immer mehr erkannt worden, und die Idee der Schulkolonie hat sich in den letzten Jahren an der Oberstufe vieler Schulen mehr oder weniger durchgesetzt. Es gibt solche, welche die Schulkolonie für eine bestimmte Klassenstufe obligatorisch erklärt haben und wiederholt dieselben bewährten Lagerorte aufsuchen. An den meisten Schulen wird die Initiative den Lehrern überlassen, so auch an der 


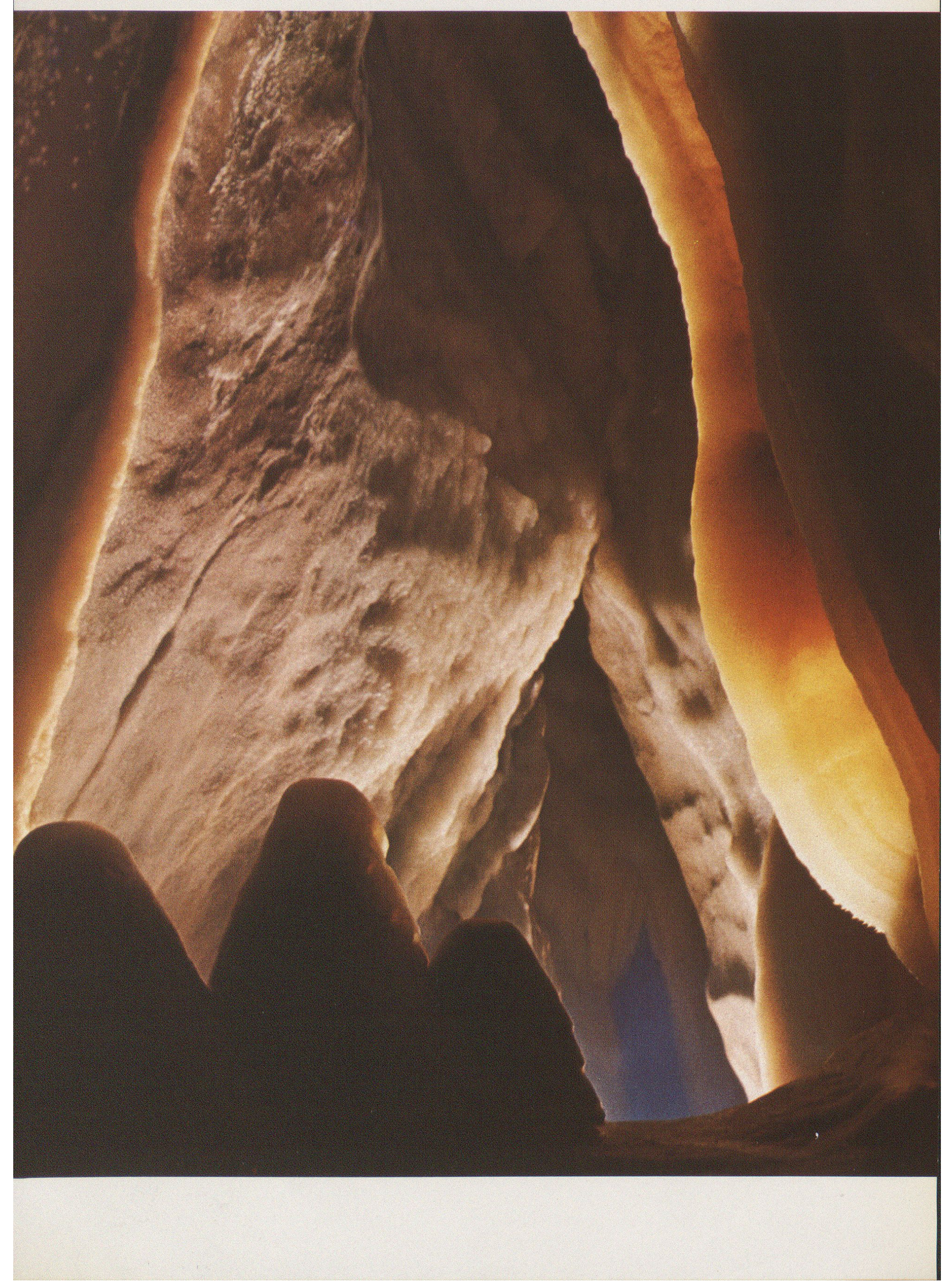


Kantonalen Handelsschule Basel. Hier besteht die Tradition, kein zweites Mal dasselbe Gebiet zu bearbeiten, was eine beträchtliche Mehrbelastung für die «lagerfreudigen» Lehrer bedeutet; dennoch werden fast jedes Jahr Kolonien durchgeführt. Selbstverständlich eignet sich nicht jede Klasse dafür, doch ist die Beurteilung der Eignung nicht einfach. Manchmal bietet eine Klasse im Studienlager ihre besten Leistungen, obwohl die Voraussetzungen in geistiger, intellektueller oder charakterlicher Hinsicht nicht günstig schienen.

Die Idee der Arbeitsgemeinschaft ist nicht nur in einem Arbeits-Studienlager realisierbar. Deshalb sei ergänzend auf einige kürzlich publizierte Beispiele hingewiesen. Im Sommer 1963 hat A. Leupin (Lit. 3) mit einer 2. Pratteler Realklasse (siebtes Schuljahr) im Rahmen der National-Reportage eine große Klassenarbeit ausgeführt und für die Monographie über Pratteln die Geographie als fächerverbindendes Fach und solide Erlebnisgrundlage für den beteiligten Sprachunterricht ins Zentrum gerückt. Besonders schöne Möglichkeiten bietet der Lehrplan des Kantonalen Gymnasiums Winterthur, der schon seit 1948 für die 6. Gymnasialklasse während des Sommersemesters fakultative halbtägige Übungen, u. a. auch in Geographie, vorsieht. Fr. Schießer (Lit. 5, S. 35) äußert sich dazu in seinem anregenden Aufsatz: «Die Bildungsziele der Schulgeographie decken sich in jeder Beziehung mit den neueren Bestrebungen gymnasialer Bildung allgemein. Dazu gehört vorerst die Konzentration des Stoffes auf Wesentliches, um Zeit für einen freieren Arbeitsstil zu gewinnen»... . «Durch Erarbeiten bestimmter Aufgaben erhält er (der Schüler) Zugang zu den wissenschaftlichen Methoden. Der Schüler soll selber in der Landschaft Zusammenhänge entdecken. Der freiere Stil kann als Vorform des akademischen Lehrbetriebes verstanden werden». Diese Formulierung paßt ausgezeichnet für den hier behandelten Schulkolonietyp, mit der Einschränkung, daß in unserm Falle Diplomanden und nicht Maturanden heranzubilden waren. Aus der von Schießer wiedergegebenen Themenliste für geographische Übungen aus sieben verschiedenen $Z$ weigen des Faches ergeben sich weitere enge Zusammenhänge mit unserer Kolonie-Idee, steht doch auch die Landschaftskunde am Beispiel kleiner Gemeinden im Programm, «damit die Bearbeitung während eines Semesters in die Tiefe gehen kann». Das oben erwähnte Thema «Pratteln» eignete sich beispielsweise wohl für eine Klassenarbeit, wäre jedoch vermutlich für eine 14-tägige Schulkolonie zu umfangreich gewesen. Gruppenübungen, wie sie in vielen Lagern üblich geworden sind, werden an fremden Orten mit Vorteil am kleinen überschaubaren Objekt durchgeführt, um die Schüler durch die Stoff-Fülle nicht zu entmutigen und ihre Selbständigkeit und Selbsttätigkeit zu gewährleisten. Bezeichnenderweise ziehen denn auch die meisten Kolonieleiter relativ kleine ländliche Gemeinden vor. Oft entstehen auf diese Weise größere oder kleinere Monographien des gewählten Gebietes, wobei die Schwergewichte je nach den Interessen der beteiligten Lehrer und Schüler mehr auf dem einen oder dem andern Fachgebiet liegen. Es wäre auch die gemeinsame Bearbeitung eines Problem-Themas möglich, aus geographischer Sicht etwa die Hochkonjunktur in den Alpen, die Gefahren der Überfremdung in ausgewählten Landschaften, die Jurafrage, Technik und Landschaftsschutz, Bedeutung und Folgen der Gewässerverschmutzung, der Kampf des Menschen gegen die Natur (Lawinenverbauungen, Aufforstungen, große Meliorationswerke) u. a. m. Allerdings eignen sich derartige Themen oft besser für die Bearbeitung in der Schule und zu Hause, wo die Literaturbeschaffung meist leichter ist. Erwähnung verdient in diesem Zusammenhang der vermutlich erste Versuch, Landesplanung an einer schweizerischen Mittelschule zu erteilen. Im Rahmen der probeweisen Einführung des Gruppenunterrichtes an der Oberschule Zürich im Jahre 1963 bot sich W. Nigg (Lit. 4) diese Möglichkeit, und zwar im Sommersemester wöchentlich während zwei aufeinanderfolgenden Stunden. Nach einführenden Literaturstudien und Kurzreferaten der Schüler sowie Diskussionen hielten kompetente Fachleute Vorträge. Im Anschluß an vorbereitende Exkursionen wurde schließlich der Versuch einer Zonenplanung von Fällanden unternommen. In dieser 
Form des Unterrichtes wird die Landesplanung zur sinnvollen Erziehungsaufgabe der Geographie im Dienste des Natur- und Heimatschutzes. Das in den genannten Beispielen zum Ausdruck kommende Streben nach zeitgemäßen Arbeitsformen findet eine schöne Ergänzung in der Arbeitsgemeinschaft einer Schulkolonie. Da in der schweizerischen Literatur zur Geographiemethodik diese Institution bisher viel zu wenig Beachtung gefunden hat, seien - vor allem als Anreiz für jüngere Kollegen - einige Anregungen aus der Praxis der Schulkolonie für die Praxis festgehalten.

\section{EINFÜHRUNG IN DIE TECHNIK DER EINZEL- UND GRUPPENARBEIT IM HINBLICK AUF DIE SCHULKOLONIE IN SEDRUN}

Das Arbeits-Studienlager der Diplomklasse D 3a in Sedrun (GR) ist eines der vielen, welche an der Kantonalen Handelsschule im Laufe der Jahre organisiert wurden. Der Schreibende zeichnete für die didaktisch-methodische Leitung, sein Kollege Dr. A. Hochuli für die technischen Belange verantwortlich. Die systematischen Vorbereitungen auf diese vom 14.-26. Juni 1965 durchgeführte Schulkolonie setzten schon 11/4 Jahre vorher ein, insbesondere aber im letzten halben Jahr, nachdem wir uns durch einen Elternabend (im Mai 1964) und die Genehmigung des Rektorates rückversichert hatten. Den definitiven Entscheid faßten wir Ende 1964, als wir uns von der Eignung der Klasse für die in Aussicht genommene Aufgabe überzeugt hatten. Hier kurz die hauptsächlichsten methodischen Vorbereitungsarbeiten in der Klasse:

- Einführung in die Geologie und Klimatologie, entsprechend dem für die Studien im Lager gewählten Stoffbereich mit Geographic als Kernfach.

- Erlernung der Technik der Einzel- und Gruppenarbeit am Beispiel Südamerikas: anschauliche Referate mit anschließender Diskussion, statistische und zeichnerische Auswertung, Förderung des kausalen Denkens (s. Lit. 2).

- Selbsttätige Buchstudien am Beispiel ausgewählter Kapitel aus der Schweizergeschichte

- Besuch des Naturhistorischen Museums: Gruppenarbeiten zur Entstehung der Landschaft von Basel und Umgebung.

- Einführung in die Technik der Litcratursuche und vorbereitende Literaturstudien der Schüler auf Grund des von mir erstellten Bücherverzeichnisses.

- Führung durch die Universitätsbibliothek: Praxis der Büchersuche und -Bestellung.

Unser Hauptanliegen bestand also in der Förderung der selbsttätigen Schülerarbeit schon lange vor dem Lager. Ohne Anleitung können unsere Schüler selten wirklich selbständig arbeiten, vor allem nicht direkt aus der Naturanschauung heraus: Die Fähigkeit zum Erkennen der Probleme muß geübt werden. Gründliche Vorarbeiten nach der Literatur vor der Kolonie und in der Kolonie, ergänzt durch die Anschauung im Gelände - beide Phasen verbunden mit Einführungen unter der Anleitung des Lehrers und mit sorgfältigen Kontrollen - scheinen uns die fruchtbarste Kombination für unsere Klassen zu sein. Die angewendete Methode des Gruppenunterrichts ermöglichte - besser als die gewöhnliche Arbeitsform der Mittelschule - auf natürliche Weise eine Einführung in das wissenschaftliche Arbeiten, indem die Schüler - mehr oder weniger selbständig - vertiefte Kenntnisse über ein neues Stoffgebiet erwarben. Es hat sich auch gezeigt, daß eine in der Schule leistungsmäßig durchschnittliche, eher sportlich als intellektuell eingestellte Klasse fähig ist, in einer Schulkolonie sehr gute Leistungen zu vollbringen. Eine psychologisch differenziertere und weniger robuste, aber vielleicht schulmäßig überdurchschnittliche Diplomklasse hätte wohl kaum bessere fachliche Leistungen erreichen können.

\section{DIDAKTISCH-METHODISCHE HINWEISE ZUR VORBEREITUNG UND DURCHFÜHRUNG DER SCHULKOLONIE}

\section{Ziel der Arbeitsgemeinschaft:}

- Studien und Erhebungen über geographische, geologische, geschichtliche, wirtschaftliche und soziale Probleme einer alpinen Landschaft; 
- Erziehung zum Teamwork in kleinen Arbeitsgruppen sowie zur Konzentration und Selbsttätigkeit;

- Durchführung der Wahlfachkurse Geländedienst und Wandern im Rahmen des Vorunterrichts (VU).

\section{Methodisches Vorgehen: Konzentrationsunterricht in Gruppenarbeit.}

- Vorbereitung in Basel: Die Themen wurden vier Monate vor Beginn der Schulkolonie bekanntgegeben und verteilt, so $\mathrm{da} B$ sich die Schüler frühzeitig mit dem selbst gewählten Stoff auseinandersetzen konnten. Die allgemeine und historisohe Literatur mußten die Schüler entsprechend dem a rbeitsunterrichtlichen Verfahren - selbst suchen und in zweckdienlicher Weise verarbeiten; das praktische Vorgehen lernten sie unter Anleitung des Deutschlehrers am Beispiel der gesuchten Bücher in der Universitätsbibliothek. Zur Entlastung der Schüler wurde die einschlägige geographische und geologische Literatur von mir gesucht und beschafft. Auch der Arbeitsplan mit den Themen enthielt zur Vermeidung unnötiger Umtriebe einige methodische Hilfen für die Schüler. Damit grobe Irrwege noch vor der Kolonie berichtigt werden konnten, mußten zwei Wochen vor der Abreise genaue Dispositionen der Arbeiten abgeliefert werden. In den letzten vier Monaten wurden durchschnittlich $1-2$ Schulstunden pro Woche für die Vorbereitung der Schüler auf ihr Kolonieprogramm verwendet, wozu noch häusliche Literaturstudien kamen.

- Arbeit in Sedrun: Die gewählte Art der Vorbereitung ermöglichte der Leitung, alle Schüler sofort, d. h. ohne Anlaufzeit einzusetzen. Eigenart und geringe Zahl der Schüler (15) ließen in dieser Klasse die Tätigkeit in Zweiergruppen, die von Fall zu Fall zu größeren Teams koordiniert werden konnten, als gegeben erscheinen. Jedes Paar bearbeitete nach Möglichkeit ein Thema nach der Literatur und ein Thema im Freien (Aufnahmen im Gelände, Besichtigungen, Interviews). Entscheidende Kriterien:

- Anschaulichkeit der Arbeiten (Skizzen, Graphica, Photos);

- Anwendung des Konzentrationsgedankens (vertieftes gemeinschaftliches Erarbeiten und Eindringen in Probleme von Natur und Volk im Tavetsch mit Betonung der Querverbindungen zwischen verschiedenen Fachgebieten);

- die Geographie als wegleitendes Kernfach neben Geschichte, Geologie, Deutsch und Französisch (rapports journaliers).

Auf diese Weise sollten die Schüler ein Gesamtbild der Landschaft und ihrer Bewohner erhalten. Neben den laufenden Gruppenarbeiten, aber stets im engsten Zusammenhang mit den im Gang befindlichen Arbeiten, wurden mit der ganzen Klasse gemeinsam einige Besichtigungen (Kraftwerkanlagen in verschiedenen Bauphasen, Kloster Disentis), Exkursionen (Touren mit Strahlern, Ausflüge und Märsche für den Wahlfachkurs Wandern) und $G c$ ländedienst (im Rahmen des VU) durchgeführt. Sie bildeten den stets willkommenen Ausgleich zu den Facharbeiten und wickelten sich nach einem speziellen Programm ab. Bedingungen und Stoffbereich der Wahlfachkurse und Wahlfachprüfungen finden sich in den Ausführungsvorschriften des Eidgenössischen Militärdepartementes über den turnerisch-sportlichen VU; wie sie ins Gesamtpensum eingegliedert wurden, ist aus dem durchgeführten Programm ersichtlich (siehe Anhang).

- Auswertung der Schülerarbeiten in der Schulkolonie: anschauliche Kurzreferate der Schüler über ihre laufenden Arbeiten, mit anschließender Diskussion (abends). In diesen gemeinsamen Arbeitsbesprechungen ist darauf zu achten, daß zwar die Referate das Gerüst einer Gesamtschau bilden, das Gespräch jedoch immer mehr in den Mittelpunkt rückt. Bei schwierigen Stoffen (etwa für geologische und mineralogische Themen) können die Referenten vor der Diskussion Stichwort-Vervielfältigungen abgeben. Jedenfalls sollen die Schüler nicht längere Zeit nur schweigen oder nur reden. Damit alle Schüler in bleibender Form über das Gesamtergebnis der Lagerarbeiten im Bilde sind, scheint es für eine Handelsschule zweckmäßig, die Reinschriften mit einem Umdrucker im Lager selbst zu vervielfältigen. Trotz verschiedenen Schwierigkeiten gelang es uns, alle technischen Arbeiten in Sedrun abzuschließen zweifellos eine erwünschte Entlastung des nachher wieder einsetzenden normalen Unterrichtsbetriebes. Außer der wechselnden Gruppe für den Inneren Dienst kann jeweils von Fall zu Fall eine technische Gruppe (Redaktoren, Vervielfältiger, Zeichner, Photographen) gebildet werden.

Soviele Vorzüge Schulkolonien aufweisen können - ein Nachteil ist unverkennbar: An eine freiere Unterrichtsform gewöhnt, haben die Sohüler zu Hause oft Mühe, in den Pensumunterricht zurückzufinden. Insbesondere müssen sie nun wieder in den im Lager nicht berücksichtigten und dabei zeitlich zu kurz gekommenen Fächern a rbeiten, vielleicht sogar nacharbeiten. Gerade deshalb sollten sämtliche Kolonieaufgaben wenn irgendwie möglich vor der Rückkehr beendigt werden. Empfehlenswert und ohne großen Zeitaufwand realisierbar ist hingegen eine 
Ausstellung im Klassenzimmer oder in einem Spezialraum (Photos, Graphica, Originalaufnahmen, Sammlungen aller Art, Werdegang der Ausarbeitung usw.).

\section{Bewertung der Schülerleistungen durch den Fachlehrer:}

Es ist gerechtfertigt, wenn der zuständige Fachlehrer (Leiter) die Gcsamtlcistung jedes Schülers, also nicht nur das vervielfältigte Endergebnis, gebührend bewertet. In unserem Fall zählte diese Fachnote etwa $1 / 3$ der Zeugnisnote des Sommersemesters. Außerdem wurde zusätzlich die sprachliche Leistung (Muttersprache, bzw. Französisch für die in der ersten Fremdsprache abgefaßten Tagesberichte) berücksichtigt. Entscheidend war in jedem Fall die Qualität und nicht die Quantität der Arbeiten.

\section{Ergebnisse und Erfahrungen:}

- Organisation und Administration: Unter dem Titel «Vorbercitung und Durchführung der Schulkolonie Sedrun 1965》 haben Kollege Dr. A. Hochuli und ich für einen kleineren Kreis ein Heft zusammengestellt, in dem die organisatorischen und administrativen Belange eines Lagers mit Selbstverpflegung erläutert werden: Rekognoszierung, Unterkunft, Reise, Fahrzeuge, Vortrupp, VU, Sicherheitsvorkehren, Verpflegung, Finanzen, Koloniebuch. Das 3o Seiten umfassende Hilfsmittel ist mit den entsprechenden Beilagen ausgestattet: Merkblatt mit provisorischem Arbeitsplan, Anmeldeformular, durchgeführtes Arbeitsprogramm, Bestellung der Unterkunft, Anforderung von Verpflegung und Material beim Büro für VU und im Kontor der Kantonalen Handelsschule, Kommandierliste, Sicherheitsvorkehren; schlieBlich enthält es drei Zusammenstellungen des Schülers, der als Rechnungsführer amtete: Menuplan, Lebensmittelliste mit Mengen und Preisen, Abrechnung. Das Beiheft möchte einen Begriff vermitteln, welche Fülle an Überlegungen, Kleinarbeit und Zeitaufwand allein schon die technische Leitung einer Schulkolonie mit Selbstverpflegung mit sich bringt.

- Methodische Erfahrungen: Es bestand der Plan, in dem im Lager fertiggestellten Beiheft auch die didaktisch-methodischen Bestrebungen zu erläutern. Aus Zeitmangel und wegen der fehlenden Distanz war die ursprüngliche Absicht in einigermaßen abgerundeter Form nicht möglich. Dieser Aufsatz möchte diese Lücke in etwas erweiterter Darstellung nachholen. Ich verzichte jedoch darauf, den zahlreichen, namentlich aus der Bundesrepublik Deutschland stammenden Gedanken zur «Methodiktheorie» einen weiteren Beitrag anzuschließen und beschränke mich darauf, das erlebte Beispiel der Schulkolonie Sedrun auszuwerten. Aus Platzgründen ist es nicht durchführbar, meinen vollständigen Arbeitsplan mit allen methodischen Anmerkungen wiederzugeben; dieses Verfahren wäre auch nicht zweckmäßig, denn der beste Arbeitsplan erfährt im Verlaufe eines Studienlagers verschiedene Änderungen. Sein Gerüst spiegelt sich im schlagwortartig kurz gehaltenen Inhaltsverzeichnis des Koloniebuches wider (siehe Anhang).

Da Maschinenschreiben, Stenographie und Kontorarbeit zum Lehrplan einer Handelsschule gehören, ist es verlockend, als Beweis für die geleistete Arbeit die Fachstudien jedem Schüler geheftet oder gar gebunden abzugeben. Der Gedanke, daß die Beiträge «veröffentlicht» werden, spornt die meisten Lagerteilnehmer an, und selbstverständlich sind sie alle stolz auf ihr Koloniebuch, für das sie sich gern den Luxus des Einbindens leisten. Die Berechnung des Materialbedarfs ist allerdings nicht einfach, und Nachbestellungen sind unvermeidlich, wenn der von der Schule mitgenommene Umdrucker durch «Ladestörungen» und andere technische Mängel den durch die Schüler verursachten Verschleiß noch steigert. Leider mußten die Leiter feststellen, daß viele Schüler dieser 3. Diplomklasse im elften Schuljahr nicht fähig waren, in einwandfreier Darstellung und sauberer Form auf den ersten Anhieb Matrizen zu schreiben und sparsam zu vervielfältigen. Allerdings stand die Klasse noch im ersten Quartal ihrer Kontorausbildung. Es zeigte sich ferner, daß eine Schreibmaschine auf zwei Schüler für diesen Kolonietyp das Minimum darstellte, vor allem deshalb, weil nur drei von sieben Maschinen die gleiche Schriftart aufwiesen. Dennoch zogen wir leicht transportable Privatgeräte den schwereren Schulmaschinen vor. Gerade die tägliche Auseinandersetzung mit den erwähnten technischen Schwierigkeiten ist eine ausgezeichnete Vorbereitung für die spätere Berufspraxis der Diplomanden! Wieviel Ausdauer und 
Energie von Schüler- und Lehrerseite notwendig sind, mag die Tatsache erhellen, daß pro Arbeit und Schüler nicht selten 2-4 Entwürfe, 1-2 Reinschriften und 1-3 Matrizen geschrieben und vom Lehrer korrigiert werden mußten - meistens willig und mehr oder weniger freiwillig, öfters in Abend- oder gar Nachtarbeit, was bei den robusten Schülern als «Plausch» galt! Im Hinblick auf den offenkundigen Arbeitseifer haben wir bei etwas einseitiger Spezialisierung der Schüler gelegentlich mehr als ein Auge zugedrückt, etwa wenn die innert 24 Stunden von der jeweiligen Küchenmannschaft in französischer Sprache verfaßten Tagesbericht zu deutlich den Stilstempel ihres zweisprachigen Klassenkameraden trugen oder wenn die guten Matrizenschreiber in der 2. Hälfte zu sehr nur schrieben, die begeisterten Zeichner zu viel nur Titelblätter entwarfen. Einige Schüler - bezeichnenderweise solche, die entweder wenig geistvolle Buchabschriften produzierten oder methodisch anspruchslosere Erlebnisberichte abfaßten gaben ihrer Enttäuschung Ausdruck, weil ich Kürzungen bis zu einem Drittel verlangte, vor allem, wenn langfädige Ausführungen oder Aufzählungen der Geschlossenheit des Themas abträglich schienen. Denn es bestand keineswegs das Ziel, möglichst viel schreiben zu lassen; auch nach den Streichungen weisen Koloniebuch und Beiheft noch den für 15 Schüler respektablen Umfang von 200 Seiten auf. Beim Gedankenaustausch sahen die anfänglich Enttäuschten ein, daß weder Seitenrekorde noch die Aufstellung eigener, aber eindeutig falscher und den vorhandenen Quellen widersprechender Thesen Endzweck der Übung sein konnten. Meine Korrekturen und selbst angebrachten Änderungen hielten sich übrigens durchaus im normalen Rahmen einer Aufsatzdurchsicht, wobei wenn immer möglich der Autor als Partner anwesend war. Nur der korrigierende Lehrer hat bei solch umfangreichen Arbeiten die Übersicht, die ihm erlaubt, die einzelnen Themen gegenseitig in ein vernünftiges Gleichgewicht zu bringen. Es wäre beispielsweise falsch gewesen, wenn bei unserer Kombination verschiedener Arbeitsformen die aus leichter Hand geschriebenen Erlebnisaufsätze die anspruchsvolleren Arbeiten im Koloniebuch überwuchert hätten. Bei jüngeren Schülern der Fachund Verkehrsabteilung mag sich das Problem wieder anders stellen. Außerdem war die von den Schülern gewünschte Behandlung geologischer und mineralogischer Aufgaben ein - allerdings vorbereiteter - Versuch, der im Interesse der Richtigkeit der Ergebnisse mühsame Kontrollen des Lehrers nach der Literatur erforderte. Unseres Erachtens darf sich das Ergebnis mit den teilweise recht anschaulichen Skizzen, Photos und Karten (zum Teil Kopien, vorwiegend aber Kombinationen und Vereinfachungen aus mehreren Quellen) neben den bisher geleisteten Arbeiten gleichstufiger Diplom- und Maturklassen sehen lassen.

$\mathrm{Zu}$ den einzelnen Themengruppen kann zusammenfassend folgendes festgehalten werden:

«Die Natur des Tavetsch»: Die immer noch ausgezeichnete Landschaftskunde des Tavetsch von Leemann war eine gesuchte und höchst brauchbare Quelle. Andrerseits mußte ich gerade bei diesem Kapitel bedeutende Kürzungen anbringen, weil einzelne Bearbeiter zuviel Abschrift und Zitat, dafür zu wenig eigene geistige Arbeit (Zusammenfassung, Beizug weiterer Bücher usw.) boten.

«Wirtschaft und Verkehr»: Da es sich vor allem um Protokolle und Interviews handelt, habe ich aus $\mathrm{Gründen} \mathrm{der} \mathrm{Originaltreue} \mathrm{auf} \mathrm{Kürzungen} \mathrm{verzichtet.}$

«Bevölkerung und Siedlung»: Außer dem einleitenden Kapitel über die Walser erfreulich selbständige Arbeiten der Schüler.

«Disentis und sein Kloster»: Im historischen und künstlerischen Teil habe ich einige Kürzungen verlangt, da Disentis nicht über das Zentralthema Tavetsch dominieren durfte.

«Geheimnisvolle Natur»: Bei den schwierigen Kapiteln über die Entstehung der Alpen und über die landschaftsgestaltenden Kräfte mußte ziemlich viel nachgeholfen werden, da es sich um einen ausgesprochen problemreichen Stoff handelt, den Schüler dieser Stufe noch nicht übersehen können. Die Mineralogie wurde bewußt von der Praxis her angegangen, und tatsächlich führte das Erlebnis der Kristallsuche mit bekannten Strahlern zu beachtenswerten Ergebnissen.

Zum Literaturverzeichnis: Bequemer ist zweifellos die Erwähnung der benutzten Quellen bei jeder Arbeit, doch diente die unter großem Zeitaufwand zusammengestellte 
und jeweils zitierte Liste als wertvolle Einführung in wissenschaftliches Arbeiten; zehn Arbeitsstunden gingen allein deshalb verloren, weil das Literaturverzeichnis nicht rechtzeitig in korrekter Ausführung vorlag.

Es braucht wohl kaum näher erläutert zu werden, daß der Klassenlehrer seine Schüler in einem 14tägigen Arbeits-Studienlager gründlicher kennenlernt, als dies in den Schulstunden, im Turnen, auf Wanderungen oder im Skilager in der Regel der Fall ist. Besonders deutlich schälten sich im Sedruner Lager einige Arbeitstypen heraus, die vermutlich in jeder ähnlich aufgezogenen Schulkolonie in mehr oder weniger ausgeprägter Art vertreten sind. Etwa der «Krampfer», der ein bienenfleißiger und kreuzbraver «Buchkopist» sein kann und viel Hilfe benötigt (mit entsprechendem Zeitaufwand für Korrekturen von Seiten des Lehrers), weil er wenig eigene Gedanken beizutragen vermag. Daneben gibt es den ausgesprochen intellektuellen Typ, der die auftauchenden Probleme selbständig durchdenkt und in Verbindung mit seinem Fleiß ausgezeichnete Resultate erreicht. Das Gegenstück zum «Krampfer» bildet der Minimalist, der die Situation der freieren Arbeitsweise in Gruppen für seine bequemen Zwecke auszunützen versucht. Er darf nur wenige Stunden sich selbst überlassen werden und muß durch häufige Kontrollen und Arbeitsbesprechungen in Fahrt gebracht werden. Eine weitere Variante stellt der rasch Arbeitende dar; bei ihm leidet unter dem Tempo oft die Qualität. Die ideale Kombination, nämlich rasch und gründlich, tritt selten auf. Häufiger trafen wir den langsamen Arbeiter, der dank seiner Gründlichkeit nach einer relativ langen Anlaufzeit ein gutes Rendement erzielt.

Zusammenfassend läßt sich feststellen, daß 7 von 15 Schülern die ihren Anlagen und Fähigkeiten und die auf Grund ihrer Schulleistungen zu erwartenden Leistungen geboten haben; gesamthaft eindeutig bessere Resultate als in der Schule erreichten 3 Lagerteilnehmer, während 3 weitere Schüler vergleichsweise eher enttäuschten. Als Ausnahmefälle auszuklammern sind der wegen Krankheit nachgereiste und noch schonungsbedürftige Schüler sowie der sehr begabte und fast überall erfolgreiche Primus, der nach dem Diplom noch die Maturität anvisieren sollte. Konstante und auffallende Einsatzbereitschaft haben 4 Schüler gezeigt, doch hat in 2 Fällen die leistungsmäßige Ausbeute nicht dem überdurchschnittlichen Fleiß entsprochen.

\section{BENÜTZTE LITERATUR}

1 Annaheim, H.: Die neuzeitliche Geographie und ihre Bedeutung für die Durchführung von Schulkolonien. Basler Schulfragen, Nr. 1, 1938. 2 Bösiger, K.: Zur Methodik des Geographieunterrichts an Handelsschulen. Geographica Helvetica, Nr. 3, 1961. 3 Leupin, A.: Pratteln, größter Industrieort von Baselland. Basellandschaftliche Schulnachrichten, H. 2, Oktober 1964. 4 Nigg, N.: Landesplanung an der Mittelschule. Geographica Helvetica, Nr. 3, 1964. 5 Schießer, Fr.: Geographische Übungen an der Oberstufe der Mittelschule. Geographica Helvetica, Nr. 1, 1965. 6 Wirth, W.: Geographische Übungen auf der Oberstufe der Mittelschule. Geographica Helvetica, Nr. 1, 1951.

\section{ANHANG}

\section{Inhaltsverzeichnis des Koloniebuches Sedrun 1965}

Vorwort der Lagerleiter

Rapports journaliers

Natur- und Menschenwerk im oberen Vorderrheintal

Die Natur des Tavetsch

Lage, Grenzen und Größe der Gemeinde Tavetsch - Untergrund, Relief und Gewässer Klimaverhältnisse und Wetterbeobachtungen - Beobachtungen zum Pflanzenkleid ob Sedrun

Wirtschaft und Verkehr im Tavetsch

Tal- und Alpwirtschaft - Handel und Gewerbe - Baugeschichte der Staumauern Nalps und Curnera - In der Kavernenzentrale Sedrun - Lukmanier- und Oberalppa $B$ 
Die Bevölkerung in der Gemeinde Tavetsch

Erschließung und Besiedlung des Tavetsch durch die Walser - Bevölkerungsentwicklung, und Volksdichte - Die heutige Gliederung der Bevölkerung nach Alter und Geschlecht - Berufstätige des Tavetsch nach Erwerbsklassen - Gliederung der Wohnbevölkerung nach dem Heimatort - Politik und Verwaltung - Besuch einer Gemeindeversammlung - Volkstum

Die Siedlung Sedrun als Hauptort der Landschaft Tavetsch

Name, Anlage und Siedlungsentwicklung des Dorfes Sedrun - Gotthardhaus und Engadinerhaus, ein Vergleich - Entstehung und Entwicklung der Pfarrei Sedrun - Die Pfarrkirche und andere Kunstdenkmäler

Disentis und sein Kloster

Geschichtliche Entwicklung der Benediktinerabtei - Zur Baugeschichte von Kirche und Kloster - Das Kloster als kulturelles Zentrum - Vom Mönchsleben in Disentis - Disentis als $\mathrm{PaBfuB-}$ - und Kurort

\section{Geheimnisvolle Natur}

Entstehung und Bau unserer Alpen - Landschaftsgestaltende Kräfte im Tavetsch - Gesteine und Mineralien im Tavetsch - Touren mit Strahlern - Besichtigung einer Kristallsammlung - Naturschutzprobleme

Literaturverzeichnis

\section{Besondere Aufgaben, Gemeinsame Exkursionen und Besichtigungen}

- Beobachtungs- und Erkundigungsaufgaben auf der Hinreise (Gruppenwettbewerb)

- Im Tagesbericht über die Hinreise behandelte Themen: Tomalandschaft zwischen Chur und Reichenau, der Flimser Bergsturz, die Sprachverhältnisse in Graubünden

- Besichtigung des Klosters Disentis (Führung)

- Besichtigung der Kraftwerkanlagen in verschiedenen Bauphasen (Führung)

- Gesteinskenntnis und Mineraliensuche mit Strahlern

- VU-Wahlfachkurs: Wandern und Geländedienst (nach speziellem Programm)

\section{Durchgeführtes Programm}

(Nachtrag zum Merkblatt mit provisorischem Arbeitsplan vom März 1965)

Der vorgesehene Arbeitsplan ist im wesentlichen programmgemäß durchgeführt worden. Infolge der vorangegangenen Schlechtwetterperiode und dem dadurch bedingten Rückstand der Schneeschmelze mußten die Touren Richtung Tomasee und Chrützlipaß im obersten Teil gekürzt werden und die für den Wahlfachkurs Wandern vorgeschriebene Marschleistung vermehrt im Bereiche des Haupttales erfüllt werden. Die übrigen Änderungen im definitiven Arbeitsplan ergaben sich aus zeitlichen Umstellungen zur Anpassung an die Verfügbarkeit unserer Gewährsleute.

Montag, 14.6.

unverändert (Gruppenarbeiten)

Dienstag, 15.6 .

Vm: unverändert (Arbeiten im Gelände)

$\mathrm{Nm}$ : Wanderung (23 km, nach VU-Berechnung) mit Besichtigung der Staumauer Nalps sowie Führung durch die Kavernen-Zentrale Sedrun.

Mittwoch, 16.6.

$\mathrm{Vm}$ : unverändert (Fortsetzung der Arbeiten, Literaturstudien, Interviews, Aufnahmen im Gelände).

Nm: Wanderung (6 km) nach Cungieri (Aussichtspunkt) und zur Drunschlucht.

Geländedienst 5/6:

Kartenlesen, Kompaßkunde, Orientierungsübungen, Ansichtskroki, Distanzenschätzen, und -messen (Geländedienst $1-4$ in Doppelstunden als Vorbereitung in der Schule vorweggenommen: gründliche Kartenkenntnis, Handhabung der Bussole, auch im Gelände).

Donnerstag, 17.6 .

Wandern: Tagesmarsch, z. T. gruppenweise, aus dem Raum Oberalppaß nach Sedrun (2o km), verbunden mit Geländedienst 7/8: Picknick: Abkochen im Freien mit Armeekochapparat (Meta): «Pot-au-feu, fixfertig»; Planskizze (Funktionsplan von Selva), Routenskizze Selva-Plaun Milez als Wegrekognoszierung, Beschreibung der am Weg liegenden Sehenswürdigkeiten. 
Freitag, 18.6.

Ganzer Tag in Disentis:

Vm: Führung durch Kloster (Zelle), Kirche und Museum mit Pater Ansgar Müller. Geländedienst 9: Picknick: Abkochen im Freien mit Armeekochapparat (Sprit) : «Berner Platte, fixfertig».

$\mathrm{Nm}$ : Wanderung $(12 \mathrm{~km})$ in die St. Agatha- und Lukmanierschlucht mit dem Strahler-Pater Flurin Maissen: Geologie des Tavetsch, Gesteinskenntnis und Mineraliensuche.

Abends: unverändert (Kurzberichte der Gruppen über den Stand der Arbeiten).

Samstag, 19.6.

Fortsetzung der Gruppenarbeiten (weitere Materialbeschaffung, Interviews, Aufnahmen im Gelände, Photographien, Verarbeitung der Ergebnisse).

Gruppe der Geologen: Tour mit zwei Strahlern ins Val Cavradi (Val Curnera) im Oberalpgebiet.

Sonntag, 2o. 6 .

Gottesdienst, Materialauswertung.

Montag, 21.6.

Vm: unverändert, Verarbeitung der Ergebnisse.

$\mathrm{Nm}$ : Geländedienst 1o: Verwendung der Zeltbahn (Zeltbau, Regenschutz usw.).

Abends: Gcländedienst 11: Repetition «Erste Hilfe» (Medikamentenkenntnis, Verbände, Tragarten, künstliche Beatmung usw.).

Dienstag, 22.6.

Auswertungen und letzte Erhebungen.

Gruppe der Geologen: Ausflug mit Strahler A. Caveng in seine neu erschlossenen Klüfte im Val Nalps; Besichtigung seiner Mineraliensammlung.

Mittwoch, 23.6.

Verarbeitung der Ergebnisse. Gedankenaustausch zwischen den Gruppen.

Donnerstag, 24.6.

Wanderung $(20 \mathrm{~km})$ ins Val Strem (Chrüzlipaß). Geländedienst 12/13: Kartenleseübungen mit LK $1: 25$ o0o und $1: 50000$.

Freitag, 25.6.

Abschlußarbeiten, Reinigen der Unterkunft, Packen.

Samstag, 26.6.

Heimreise via Oberalp-Göschenen-Luzern.

\section{LA GEOGRAPHIE DANS LES COLONIES SCOLAIRES; UNE DISCIPLINE DE SYNTHESE ET DE COORDINATION}

Ce n'est qu'au cours des dernières décennies que l'idée des camps d'études s'est lentement imposée dans la division supérieure de nombreuses écoles du second degré. La valeur inestimable de telles communautés de travail est aujourd'hui indiscutable. La littérature consocrée en Suisse aux questions de méthode dans le domaine de la géographie n'a pas encore prêté à l'institution des colonies scolaires l'attention à laquelle celle-ci a droit. C'est pourquoi l'auteur de cet article voudrait tirer de l'expérience qu'il a faite à Sedrun (Grisons) avec une classe finale de troisième de l'Ecole cantonale de Commerce de Bâle (onzième année scolaire), quelques suggestions qui pourraient stimuler l'intérêt de collègues plus jeunes pour ce problème.

Une importance particulière a été accordée à la préparation adéquate des élèves par une introduction minutieuse à la technique du travail individuel et à celle du travail de groupe. C'est du but que s'est proposé la communauté que découle en grande partie la voie méthodique à suivre, pendant la colonie et, le cas éohéant, après celle-ci. Les expériences, pour leur plus grande part positives, faites pendant le camp sont illustrées par quelques exemples. En appendice, on a reproduit le sommaire du livre de la colonie, tel qu'il a été rédigé dans le camp et polygraphié ensuite sous sa forme définitive, ainsi que le programme parcouru. 